\title{
An exemplar-based learning model for hydrosystems prediction and categorization
}

\author{
Fi-John Chang*, Li Chen \\ Department of Agricultural Engineering, National Taiwan University, 1, Sec. 4, Roosevelt Rd., \\ Taipei, 10770, Taiwan
}

Received 8 February 1994; accepted 7 September 1994

\begin{abstract}
The main purpose of this paper is to represent a new Exemplar-Based Learning model and to apply this model for river flow estimation. The central idea of the model is based on a theory of learning from examples. This idea is similar to human intelligence: when people encounter new situations, they often explain them by remembering old experiences and adapting them to fit.

To explore the stability and efficiency of the model performance, a simple mathematical function is simulated by the model. The model is then applied to extend the annual stream flow records according to the nearby stream flow stations and to classify the monthly flow by using the monthly rainfall and runoff information in the previous months. The results show that the model has better performance than the traditional methods and the results demonstrate the power and efficiency of the model for the hydrological data analysis.
\end{abstract}

\section{Introduction}

Natural phenomena are marvellously complex and varied, and events never repeat exactly. But even though events are never the same, they are also not completely different. There is a thread of similarity and continuity, so we are able to predict from past experience to future events. Although natural phenomena in general do not have well-defined rules or fixed boundaries separating alternatives, most early work in model fitting used well-defined concepts, such as regression analysis or hypothesis testing theories. Focusing on such issues has the relative difficulty of acquiring different rulcs, strategics for testing alternative hypothesis, and the transfer of behavior to new stimulus sets.

\footnotetext{
* Corresponding author.
} 
Owing to recent technological advances in computer-aided design, it is now much easier to build massively parallel machines. This has contributed to a new wave of interest in models of computation that are inspired by neural nets rather than the formal manipulation of symbolic expressions. The exemplar-based learning (EBL) algorithm is one of the new trends in that domain. The EBL is a very powerful method for category formation, especially in complex real-world situations where complete causal information is not available. The basic idea of this algorithm is based on a theory of learning from examples. This idea is similar to much of human intelligence. When people encounter a new situation, they often explain it by remembering old experiences and adapting them to fit.

In this paper we describe the nested hyper-rectangles learning model (Salzberg, $1988,1989,1991$ ) which is based on the EBL algorithm (Medin and Schaffer, 1978; Schank and Leake, 1989; Minton et al., 1989). Next, we explore the stability and efficiency by using the nested hyper-rectangles learning model to: (1) simulate a simple mathematical function; (2) extend the short record stream fiow; (3) classify the monthly flow. We then conclude by comparing the results from the nested hyperrectangles learning model with traditional approaches.

\section{Major characteristics of the exemplar-aided constructor of hyper-rectangles (EACH)}

The strategy of the exemplar-based learning (EBL) algorithm is based on storing points (or examples) in Euclidean $n$-space, $E^{n}$, where $n$ is the number of variables or features in an example, then comparing the new example to those, and finding the most similar example in memory. The algorithm is a very powerful tool for prediction or categorization tasks. The EACH, proposed by Salzberg (1989), is a new theory of EBL where the points in the EBL are generalized into hyper-rectangles. As the generalizations grow large, there may exist some exceptions which create 'holes' in the hyper-rectangles. These may in turn give holes inside them, resulting in a nested structure of hyper-rectangles.

To show the powerfulness and usefulness of the EACH, several important characteristics summarized from Salzberg (1989) are described below.

\subsection{Knowledge representation schemes}

Many systcms acquire rulcs during their learning processes; however, these rules do not exhaust the possible representation of the knowledge that learning may acquire. $\mathrm{EACH}$, on the other hand, creates a memory space filled with exemplars, some of which represent generalizations; and some of which represent individual examples from the system's experience.

\subsection{Learning strategies}

Incremental and non-incremental learning are two main strategies used by machine learning systems (Michalski et al., 1983). The main shortcoming of non-incremental 
strategies is inefficiency; for example, to insert a new example, the entire set of previous examples must be added to build a model. EACH is an incremental learning strategy in which systems model with each example they process; however, it also has a disadvantage in that it is sensitive to the order of the inputs. As stated by Salzberg (1991) the problem of creating an optimal number of rectangles to classify a set of points is NP-hard, consequently the problem can not be solved in polynomial time now.

\subsection{One-shot learning}

A machine learning system capable of one-shot learning should be able to learn a new concept or category from one example. Some learning models rely on notions of statistics which make one-shot learning very difficult. Some systems require that statistical outliers be confirmed by additional examples before they can be used in category formation. In EACH, once a new point has been stored in memory, which may occur after a single example, it is immediately available for use in prediction.

\subsection{Discrete and continuous variables}

EACH can handle variables that are binary, discrete, and/or continuous. For continuous variables, the model has an error tolerance parameter, which indicates how close two values must be in order to be treated as 'match'; consequently, the continuous variables are approximated by a discrete set of values.

\subsection{Disjunctive concepts}

Many concept learning programs have ignored disjunctive concepts because they are more difficult to learn than conjuncts. EACH handles disjunctions very easily in its structured exemplar memory. It can store many distinct exemplars which carry the same category label. A new example which matches any one of these distinct exemplars will fall into the category they represent. A set of such exemplars represents a disjunctive concept definition.

Salzberg (1989) used the EACH on three different domains: predicting the recurrence of breast cancer, classifying iris flowers, and predicting survival times for heart attack patients. In all cases, he demonstrated that EACH performs as well as, or better than, other algorithms which were run with the same data sets.

\section{The nested generalized exemplar learning algorithm}

The basic algorithm of EACH is that it uses some existing events as a foundation to predict the outcomes of other events by building the structure of hyper-rectangles. This process includes adjusting the weights of the model's parameters in time and making the system learn. The main procedures are described as below. 


\subsection{Initial work}

For the sake of making predictions, EACH must have a history of examples as the bases of its predictions. Consequently, the first step is to seed some examples which are randomly chosen from the training set. This process simply stores each selected example in memory without attempting to make any predictions. An example, in fact, is a vector of features, where each feature may have any number of values, ranging from 2 (for binary variables) to infinity (for real valued). According to Salzberg (1991), the number of seeds was determined by trial and error on a simulated data set, and performance was insensitive to the size of the seed set.

\subsection{Match and classify}

After initialization, every new example is matched to memory by a matching process. This process uses the distance metric to measure the distance (or similarity) between a new data point (an example) and an exemplar (an existing point or hyper-rectangle in $E^{n}$ ). The best match is the one which has the smallest distance and is then used to make a prediction in the way that the new example has the same category as the closest matching exemplar.

Let the new example be $E$ and the existing hyper-rectangle be $H$. The match score between $E$ and $H$ is calculated by measuring the Euclidean distance between the two objects. The distance is determined as follows:

$$
D_{E H}=W_{h} \sqrt[2]{\sum_{i=1}^{m}\left(W_{i} \frac{E_{\mathrm{f} i}-H_{\mathrm{f} i}}{\max _{i}-\min }\right)^{2}}
$$

Let $H_{\text {lower }}$ be the lower end of the range, and $H_{\text {upper }}$ be the upper end, then the distance metric becomes

$$
E_{\mathrm{f} i}-H_{\mathrm{f} i}=\left\{\begin{array}{l}
E_{\mathrm{f} i}-H_{\text {upper }} \text { when } E_{\mathrm{f} i}>H_{\text {upper }} \\
H_{\text {lower }}-E_{\mathrm{f} i} \text { when } E_{\mathrm{f} i}<H_{\text {lower }} \\
0 \text { otherwise }
\end{array}\right.
$$

The distance measured by this formula is equivalent to the length of a line dropped perpendicularly from the point $E_{f i}$ to the nearest surface, edge, or corner of $H$. Note that points internal to a hyper-rectangle have distance 0 to that rectangle.

Where $W_{h}$ is the weight of the exemplar $H, W_{i}$ is the weight of the featurc $i, E_{\mathrm{f} i}$ is thc value of the $i$ th feature in example $E, H_{\mathrm{f} i}$ is the value of the $i$ th feature in exemplar $H$, $\min _{i}, \max _{i}$ are the minimum and maximum values of that feature, and $m$ is the number of features.

The formula divides distances along each dimension by $\left(\max _{i}-\min _{i}\right)$ in order to standardize them to the interval $[0,1]$ so that each feature in the exemplars has the same basis.

There are two weights in the distance metric, $W_{h}$ and $W_{i} . W_{h}$ is a simple measure of how frequently the exemplar, $H$, has been used to make a correct prediction. $W_{h}$ is the ratio of the number of times $H$ has been used to the number of times it has resulted in 
a correct prediction. Consequently, it can indicate the reliability of each exemplar. Thus if a hyper-rectangle $H$ is used many times, but nearly always makes the wrong prediction, the weight $W_{h}$ will grow very large, and $H$ will tend not to be chosen as the closest match in the future. If $H$ is a noisy point, then it will eventually be ignored as $W_{h}$ increases. The minimum value of $W_{h}$ is unity in the case of perfect prediction.

The other weight measure, $W_{i}$, is the weight of the $i$ th feature. These weights are adjusted to reflect the fact that all features do not normally have equal importance in a category decision.

\subsection{Feedback}

EACH adjusts the weights $W_{i}$ on the feature fi after discovering that it has made the wrong prediction. Weight adjustment is executed in a very simple loop: for each $\mathrm{f} i$ if $E_{\mathrm{fi}}$ matches $H_{\mathrm{fi}}$, the weight $W_{i}$ is increased by setting $W_{i}=W_{i}(1+\Delta f)$, where $\Delta f$ is the global feature adjustment rate. An increase in weight causes the two objects to seem farther apart; if $E_{\mathrm{f} i}$ does not malch $H_{\mathrm{f} i}$, then $W_{i}$ is decreased by setting $W_{i}=W_{i}(1-\Delta f)$.

\subsection{The procedures of $\mathrm{EACH}$}

Fig. 1 is the flow chart of the EACH algorithm. The procedure is summarized as follows.

(1) Seeding: EACH uses historical examples to predict events in the future.

(2) Matching: use a distance metric. Each new example is matched to the exemplar in the memory with the minimum distance score.

(3) Feedback:

(a) if the system makes a correct prediction, then a new hyper-rectangle is generalized;

(b) if the system makes a wrong prediction, then the new example $E$ is taken as a single point and stored in the memory.

(4) Learning: When the system makes a mistake, $\mathrm{EACH}$ can automatically adjust each $W_{i}$ in the following way:

(a) if any $E_{\mathrm{fi}}$ is too close to $H_{\mathrm{fi}}$, then $W_{i}$ is set to $W_{i}(1+\Delta f)$;

(b) if any $E_{\mathrm{f} i}$ is not close to $H_{\mathrm{f} i}$, then $W_{i}$ is set to $W_{i}(1-\Delta f)$.

\subsection{Explanation of paradigm}

Fig. 2 shows the geometric picture of the seeding and matching processes for the case of two variables.

Let function: $\mathrm{Y}=3 \mathrm{X} 1+2 \mathrm{X} 2$ and error tolerance $=6$

Given: three training data $=(2,1 ; 8),(3,2 ; 13),(1,7 ; 17)$

Find: two predicting data $=\left(5,6 ; \mathrm{Y}_{\mathrm{P} 1}\right),\left(2,2 ; \mathrm{Y}_{\mathrm{P} 2}\right)$ 




Fig. 1. The flow chart of the EACH algorithm.

1. Training:

(0) seeding one point:

input: $\mathrm{H} 1(2,1), \mathrm{Y}_{\mathrm{H} 1}=8$

memorize one point $[(2,1) ; 8]$

(1) adding one point

input: $\mathrm{E} 1(3,2), \mathrm{Y}_{\mathrm{E} 1}=13$

calculatc: $\mathrm{D}_{\mathrm{E} 1 \mathrm{H} 1}=\left[(3-2)^{2}+(2-1)^{2}\right]^{0.5}=1.414$

$$
\text { error }=\left|Y_{E 1}-Y_{E 2}\right|=|13-8|=5<6
$$

execute: construct a hyper-rectangle $\mathrm{H} 1$

memorize: a hyper-rectangle $[(2,1),(3,2) ; 8]$

(2) adding another point

input: $\mathrm{E} 2(1,7), \mathrm{Y}_{\mathrm{E} 2}=17$

calculate: $\mathrm{D}_{\mathrm{E} 2 \mathrm{H} 1}=\left[(1-2)^{2}+(7-2)^{2}\right]^{0.5}=5.099$

$$
\text { error }=\left|\mathrm{Y}_{\mathrm{E} 2}-\mathrm{Y}_{\mathrm{H} 1}\right|=|17-8|=9>6
$$

execute: store a single point E2

memorize: a hyper-rectangle $[(2,1),(3,2) ; 8]$ and a point $[(1,7) ; 17]$ 




Fig. 2. The geometric picture for the EACH.

2. Predicting:

(1) $\mathrm{P} 1(5,6), \mathrm{Y}_{\mathrm{P} 1}=$ ?

input: $(5,6)$

calculate: $\mathrm{D}_{\mathrm{P} 1 \mathrm{H} 1}=\left[(5-3)^{2}+(6-2)^{2}\right]^{0.5}=4.472$

$\mathrm{D}_{\mathrm{P} 1 \mathrm{E} 2}=\left[(5-1)^{2}+(6-7)^{2}\right]^{0.5}=4.123$

execute: match point $\mathrm{E} 2$

$$
\text { set } Y_{P 1}=Y_{E 2}=17 \text { (true value of } Y_{P 1}=20 \text { ) }
$$

(2) $\mathrm{P} 2(2,2), \mathrm{Y}_{\mathrm{P} 2}=$ ?

input: $(2,2)$

calculate: $\mathrm{D}_{\mathrm{P} 2 \mathrm{H} 1}=0(\mathrm{P} 2$ within hyper-rectangle $\mathrm{H} 2)$

$$
\mathrm{D}_{\mathrm{P} 2 \mathrm{E} 2}=\left[(2-1)^{2}+(2-7)^{2}\right]^{0.5}=5.099
$$

execute: match hyper-rectangle $\mathrm{H} 1$

set $Y_{P 2}=Y_{H 1}=8$ (true value of $\left.Y_{P 2}=10\right)$

\section{Tests, results and discussions}

\subsection{Theoretical case}

Stability and efficiency are two important factors when one deals with model performance. To show the general characteristics of the EACH performance, a simple mathematical function,

$$
Y=X 1^{2}+X 2^{2} \quad(0 \leqslant X 1, X 2 \leqslant 1)
$$

is simulated by the model where the independent variables $X 1$ and $X 2$ are used to 
Table 1

Relationship between the number of hyper-rectangles and predictive accuracy

\begin{tabular}{lll}
\hline $\begin{array}{l}\text { The number of } \\
\text { hyper-rectangles }\end{array}$ & Mean (\%) & Standard deviation (\%) \\
\hline 5 & 55.94 & 15.02 \\
10 & 78.00 & 11.29 \\
15 & 85.72 & 8.59 \\
20 & 90.78 & 5.58 \\
25 & 94.45 & 2.72 \\
\hline
\end{tabular}

predict the dependent variable $Y$. First, an $n$ set of $(X 1, X 2, Y)$ is randomly selected as input data for $\mathrm{EACH}$. The function has two random variables, $X 1$ and $X 2$, so the distance metric is two dimensions. The error tolcrance is set as 0.2 ; i.c. if the difference between the forecasting value and true value is within 0.2 , it is considered as a 'match' and is set in the same hyper-rectangle. The procedure then is run with all the $n$ data set to build up the nested hyper-rectangles in the $E^{2}$ domain.

After the training task is completed, the EACH model's performance is then estimated. It begins with randomly selected $N$ new sets of $(X 1, X 2)$, then uses the set model, without modifying or changing the model structure or parameters, to predict $Y$ values and compare the predicted values with true values from the mathematical function. If the error is within 0.2 , the forecast result is accounted as correct; otherwise it is incorrect. In order to determine the effect of the number of nested hyper-rectangles, $m$, to the prediction accuracy, we test several different numbers of $m$, i.e. $m=5,10,15,20,25$. In cach casc, 100 sets of $(X 1, X 2)$ were performed to match their $Y$ values by using the EACH model with a different number of nested hyper-rectangles in the model, and the number of correct times were recorded. The procedures were run 100 times for the purpose of statistical analysis.

The results are shown in the Table 1 . We found that as ' $m$ ' increases, the mean value of the correct times will increase and the standard deviation will decrease. The results support the concept that when we have more experiences, we are more sophisticated.

\subsection{Stream flow extension}

Stream flow records have been extensively used in a wide variety of water resources studics. Unfortunatcly, we often face the problem that the records of stream flow are too short to contain a sufficient range of hydrological conditions or have periods of missing data. To solve this problem, we may transfer information from nearby stream gages, that is, use the historic records and extend them in time by the correlation between flow at the site of interest and concurrent flow at nearby gage(s) (Alley and Burns, 1983). However, if the correlation is low, extending the records by using such a method directly would be questionable.

The nested hyper-rectangles algorithm provides a feasible alternative in this circumstance. In the following, we used four stream flow gages in the Potomac basin, USA to demonstrate the usefulness of the algorithm for annual stream flow extension. 
Table 2

Mean annual flow in c.f.s. at the Strausburg, Antietam, Point of Rocks and Cumberland stations (19311960)

\begin{tabular}{|c|c|c|c|}
\hline Strausburg (SI) & Antietam (S2) & $\begin{array}{l}\text { Point of } \\
\text { Rocks (S3) }\end{array}$ & Cumberland (S4) \\
\hline 282 & 158 & 4642 & 731 \\
\hline 675 & 309 & 10100 & 1314 \\
\hline 344 & 268 & 7767 & 1192 \\
\hline 325 & 268 & 7056 & 1344 \\
\hline 804 & 242 & 11350 & 1649 \\
\hline 330 & 127 & 4665 & 643 \\
\hline 742 & 383 & 10840 & 1237 \\
\hline 741 & 479 & 11010 & 1336 \\
\hline 745 & 423 & 13030 & 1609 \\
\hline 500 & 278 & 8543 & $123 \mathrm{I}$ \\
\hline 1047 & 378 & 13210 & 1440 \\
\hline 490 & 228 & 7867 & 1228 \\
\hline 241 & 192 & 5220 & 750 \\
\hline 592 & 257 & 8828 & 1172 \\
\hline 567 & 231 & 8925 & 1439 \\
\hline 366 & 181 & 6849 & 1134 \\
\hline 998 & 357 & 13480 & 1652 \\
\hline 461 & 242 & 6744 & 986 \\
\hline 430 & 270 & 7317 & 1087 \\
\hline 545 & 280 & 9108 & 1175 \\
\hline 610 & 251 & 9002 & 1113 \\
\hline 494 & 209 & 8692 & 1218 \\
\hline 769 & 336 & 12670 & 1574 \\
\hline 984 & 340 & 13440 & 1570 \\
\hline 767 & 251 & 10670 & 1356 \\
\hline 226 & 173 & 4856 & 760 \\
\hline 790 & 344 & 12700 & 1480 \\
\hline 378 & 168 & 6920 & 1060 \\
\hline 232 & 125 & 4920 & 852 \\
\hline 402 & 229 & 6490 & 799 \\
\hline
\end{tabular}

The data of annual stream flows from 1931 to 1960 were obtained from Salas et al. (1980) and are shown in Table 2. For each case, one station was taken as having a short-record gage so that its last 10 years' annual flow needed to be estimated and the other three stations were taken as having a long-record. The first 20 years of concurrent annual flow of the short-record gage and the long-record gages were used as a training set to build up the EACH model. As the model is set, its structure and parameters would not be modified and it is used to predict directly the last 10 years' stream flow for the short record gage by using the concurrent records from the other three gages. The error tolerance is set to 85 c.f.s. For comparison, the multiple linear regression is performed with the same data set for each case. The results are shown in Table 3. The mean absolute error of $\mathrm{EACH}$ is smaller than that of multiple regression even in the case where it has a very high correlation. Apparently, the EACH has better performance than the multiple regression. 
Table 3

The mean absolute errors of four stations using EACH and multiple regression methods

\begin{tabular}{lcc}
\hline Names of station & Hyper-rectangle & Linear regression \\
\hline Strausburg (S1) & 58.7 & 63.2 \\
Antietam (S2) & 43.8 & 53.8 \\
Point of Rocks (S3) & 488.2 & 537.5 \\
Cumberland (S4) & 63.9 & 128.6 \\
\hline
\end{tabular}

Years of training, $N=20$; years of prediction, $m=10$.

The function of linear regression and the coefficients of multiple determination: $\mathrm{S} 1=1.153-0.698 \mathrm{~S} 2+$ $0.13 \mathrm{~S} 3-0.325 \mathrm{~S} 4, \quad R^{2}=0.9345 ; \quad \mathrm{S} 2=56.339-0.397 \mathrm{~S} 1+0.078 \mathrm{~S} 3-0.204 \mathrm{~S} 4, \quad R^{2}=0.7517 ; \quad \mathrm{S} 3=$ $-310.522+6.343 \mathrm{~S} 1+6.716 \mathrm{~S} 2+3.051 \mathrm{~S} 4, \quad R^{2}=0.9768 ; \quad \mathrm{S} 4=268.44-1.14 \mathrm{~S} 1-1.258 \mathrm{~S} 2+0.22 \mathrm{~S} 3$, $R^{2}=0.8488$.

In the following it is shown how the number of training sets influences the prediction; the gage at Point of Rocks (S3) is used as a case study. The first 5, 10, 15 and 20 years of stream flow records are used to set up the EACH model. The model is then used to predict the last 10 years' stream flows. The multiple linear regression is also performed by using the same data sets in each situation. The results are shown in Table 4.

The results are consistent with the theoretical case; that is, the model has better performance as the training data are increased. Also, the EACH has smaller mean absolute errors than the multiple regression in most of the cases, even the regression correlation is very high. Moreover, it is found that the mean absolute error in the linear regression case for $N=5$ is 815 , while for $N=10$ it is 1030 and for $N=15$ it is

Table 4

The results of station Point of Rocks (S3) as $N=5,10,15,20$

\begin{tabular}{|c|c|c|c|c|c|c|c|c|}
\hline \multirow[t]{2}{*}{ Flows } & \multicolumn{2}{|l|}{$N=5$} & \multicolumn{2}{|l|}{$N=10$} & \multicolumn{2}{|l|}{$N=15$} & \multicolumn{2}{|c|}{$N=20$} \\
\hline & Linear & Hyper & Linear & Hyper & Linear & Hyper & Linear & Hyper \\
\hline 9002 & 7717 & 10100 & 8019 & 8543 & 8007 & 8828 & 8640 & 8828 \\
\hline 8692 & 8257 & 7767 & 7194 & 8543 & 8303 & 7867 & 7943 & 7867 \\
\hline 12670 & 12483 & 11350 & 12841 & 13030 & 11663 & 13030 & 11626 & 13030 \\
\hline 13440 & 12345 & 11350 & 13207 & 11350 & 11479 & 13210 & 13004 & 13210 \\
\hline 10670 & 9707 & 10100 & 9390 & 10100 & 9368 & 10100 & 10377 & 10100 \\
\hline 4856 & 4108 & 4642 & 3631 & 4642 & 5312 & 5220 & 4604 & 5220 \\
\hline 12700 & 11742 & 11350 & 12640 & 13030 & 11148 & 13030 & 11526 & 13030 \\
\hline 6920 & 6540 & 7767 & 5129 & 7767 & 6977 & 7767 & 6449 & 6849 \\
\hline 4920 & 4391 & 4642 & 2728 & 4642 & 5346 & 5220 & 4600 & 5220 \\
\hline 6490 & 4917 & 4642 & 5621 & 4642 & 6009 & 4642 & 6215 & 4642 \\
\hline Mean absolute error & 815 & 1054 & 1030 & 715 & 863 & 585 & 537 & 488 \\
\hline
\end{tabular}

The function of linear regression: $N=5, \mathrm{~S} 3=-4119.914-0.675 \mathrm{~S} 1+10.558 \mathrm{~S} 2+8.624 \mathrm{~S} 4 ; N=10, \mathrm{~S} 3=$ $-5199.247+1.268 \mathrm{~S} 1+28.205 \mathrm{~S} 2+4.821 \mathrm{~S} 4 ; \quad N=15, \mathrm{~S} 3=-1115.325-0.948 \mathrm{~S} 1+11.092 \mathrm{~S} 2+6.214 \mathrm{~S} 4$ $N=20, \mathrm{~S} 3=-310.522+6.343 \mathrm{~S} 1+6.716 \mathrm{~S} 2+3.051 \mathrm{~S} 4$. 
863 , i.e. the addition of $200 \%$ extra training points has worsened the performance of the linear regression method. This anomaly is due to small sample size and inconsistent data structures within the training sets and predicting years.

\subsection{Stream flow forecasting}

The final task in this series given to $\mathrm{EACH}$ was to classify/predict the average daily stream flow in April for 29 years at the Shihmen Reservoir, Taiwan. The data sets were obtained from Chang and Hsu (1990) and they also cover three different hydrological factors which may influence the stream flow in April: (1) the average daily stream flow; (2) the maximum daily stream flow; (3) the average daily rainfall in the. Each stream flow was classified as three levels as obtained from Chang and $\mathrm{Hsu}$ (1990). The level of stream flow $(Y)$ was classified as follows. If:

(1) $Y \leqslant 9$ CMS then stream flow under average $(y 1)$;

(2) $9<Y \leqslant 16$ CMS then average condition $(y 2)$;

(3) $16<Y \leqslant 50$ CMS then stream flow above average $(y 3)$.

EACH seeded the 29 years' records. After the training process, it established 12 hyper-rectangles and it could precisely predict the level of $Y$ by using the three hydrological factors in the previous month (i.e. March). Comparing the optimal calibrated results obtained with Baye's decision rules and fuzzy inference rules (with the same data set) obtained from Chang and Hsu (1990), showed that the correct prediction rates were $51.7 \%$ for Baye's rules and $62.1 \%$ for fuzzy inference rules, while the EACH could make a perfect prediction. The fuzzy inference rules and EACH were also used to predict the average daily stream flow of April in 1986 and 1987, and both methods made a correct prediction. The results are shown in Table 5.

\section{Conclusion}

Three advantages of the nested hyper-rectangle learning model have been found.

(1) Saving computer memory: similar types of data are categorized as a hyperrectangle and only two diagonal points of each are memorized in its computing procedure, so the memory space can be reduced dramatically.

(2) Learning ability: the model can dynamically adjust its parameters through the feedback of new added examples. The model has the ability to learn. Consequently, the model will be more sophisticated for prediction and/or categorization if more examples (data) have been used on its training procedure.

(3) Fast execution: because the executing time is linear (not exponential) to the number of hyper-rectangles; so even if there are thousands of hyper-rectangles, the result can be obtained within a few seconds.

The model is applied to extend annual stream flows using the long records of nearby gage-stations. The results show that the model is better than the multiple linear regression method. In the case of classifying the monthly flow, the model 
Table 5

Streamflow classification /prediction using three different methods

\begin{tabular}{|c|c|c|c|c|c|c|c|c|}
\hline \multirow{2}{*}{$\frac{\text { Year }}{1957}$} & \multirow{2}{*}{$\frac{\text { Flow }}{17.3}$} & \multirow{2}{*}{$\frac{\text { Class }}{y 3}$} & \multicolumn{2}{|c|}{ Baye's } & \multicolumn{2}{|c|}{ Fuzzy } & \multicolumn{2}{|c|}{ Hyper } \\
\hline & & & $y 2$ & - & $y 3$ & + & $y 3$ & + \\
\hline 1958 & 9.4 & $y 2$ & $y 2$ & + & $y 2$ & + & $y 2$ & + \\
\hline 1959 & 17.2 & $y 3$ & $y 1$ & - & $y 1$ & - & $y 3$ & + \\
\hline 1960 & 12.7 & $y 2$ & $y 2$ & + & $y 1$ & - & $y 2$ & + \\
\hline 1961 & 11.8 & $y^{2}$ & $y 3$ & - & $y^{3}$ & - & $y 2$ & + \\
\hline 1962 & 22.6 & $y 3$ & $y 1$ & - & $y 3$ & + & $y 3$ & + \\
\hline 1963 & 5.6 & $y 1$ & $y 1$ & + & $y 1$ & + & $y 1$ & + \\
\hline 1964 & 6.1 & $y 1$ & $y 1$ & + & $y 1$ & + & $y 1$ & + \\
\hline 1965 & 6.7 & $y 1$ & $y 1$ & + & $y 1$ & + & $y 1$ & + \\
\hline 1966 & 9.4 & $y 2$ & $y 2$ & + & $y^{2}$ & + & $y 2$ & + \\
\hline 1967 & 7.0 & $y 1$ & $y 1$ & + & $y 1$ & + & $y 1$ & + \\
\hline 1968 & 26.7 & $y 3$ & $y 3$ & + & $y 3$ & + & $y 3$ & + \\
\hline 1969 & 8.3 & $y 1$ & $y 2$ & - & $y 2$ & - & $y 1$ & + \\
\hline 1970 & 12.5 & $y 2$ & $y 2$ & + & $y 3$ & - & $y 2$ & + \\
\hline 1971 & 8.2 & $y 1$ & $y 1$ & + & $y 1$ & + & $y 1$ & $t$ \\
\hline 1972 & 10.0 & $y 2$ & $y 1$ & - & $y 1$ & - & $y 2$ & + \\
\hline 1973 & 15.5 & $y 2$ & $y 1$ & - & $y 1$ & - & $y 2$ & + \\
\hline 1974 & 24.5 &.$y 3$ & $y 2$ & - & $y 2$ & - & $y 3$ & + \\
\hline 1975 & 22.0 & $y 3$ & $y 2$ & - & $y 3$ & + & $y 3$ & + \\
\hline 1976 & 8.7 & $y 1$ & $y 2$ & - & $y 1$ & + & $y 1$ & + \\
\hline 1977 & 4.6 & $y 1$ & $y 1$ & + & $y 1$ & + & $y 1$ & + \\
\hline 1978 & 33.0 & $y 3$ & $y 3$ & + & $y 3$ & + & $y 3$ & + \\
\hline 1979 & 14.4 & $y 2$ & $y^{2}$ & + & $y 2$ & + & $y 2$ & + \\
\hline 1980 & 10.3 & $y 2$ & $y 1$ & - & $y^{2}$ & + & $y 2$ & + \\
\hline 1981 & 8.8 & $y 1$ & $y^{2}$ & - & $y^{3}$ & - & $y 1$ & + \\
\hline 1982 & 9.7 & $y 2$ & $y 3$ & - & $y 3$ & - & $y 2$ & + \\
\hline 1983 & 36.8 & $y 3$ & $y 3$ & + & $y 3$ & + & $y^{3}$ & + \\
\hline 1984 & 22.1 & $y 3$ & $y^{2}$ & - & $y 2$ & - & $y 3$ & + \\
\hline 1985 & 31.0 & $y 3$ & $y 3$ & + & $y 3$ & + & $y 3$ & + \\
\hline \multicolumn{3}{|c|}{ Accuracy $(\%)$} & \multicolumn{2}{|c|}{51.7} & \multicolumn{2}{|c|}{62.1} & \multicolumn{2}{|l|}{100} \\
\hline
\end{tabular}

+ , Correct classification/prediction.

-, Incorrect classification/prediction.

does completely distinguish the investigated data set, while the rates of correct classification of Baye's decision rules and fuzzy inference rules are $51.7 \%$ and $62.1 \%$, respectively, which were obtained from Chang and Hsu (1990). The results demonstrate that the model is a very powerful and efficient tool for prediction and/or categorization in the field of hydrological data analysis.

\section{References}

Alley, W. M. and Burns, A. W., 1983. Mixed-station extension of monthly streamflow records. ASCE J. Hydraul. Eng., 109(10): 1272-1284.

Chang, F.J. and Hsu., K.L., 1990. A study for streamflow forecast. J. Chin. Agric. Eng., 36(4): 1-12.

Medin, D. and Schaffer, M., 1978. Context theory of classification learning. Psychol. Rev., 85(3): 207-238. 
Michalski, R., Carbonell, J. and Mitchell, T. (Editors), 1983. Machine Learning. Springer, Berlin.

Minton, S., Carbonell, J.G., Knoblock, C.A., Kuokka, D.R., Etzioni, O. and Yolanda, G., 1989. Explanation-based learning: a problem solving perspective. Artif. Intell., 40: 63-118.

Salas, J.D., Delleur, J.W. and Lane, W.L., 1980. Applied Modeling of Hydrologic Time Series. Water Resources, Littleton, CO.

Salzberg, S., 1988. Exemplar-based learning: theory and implementation. Tech. Rep. TR-10-88, Center for Research in Computing Technology, Harvard University.

Salzberg, S., 1989. Nested Hyper-Rectangles for Exemplar-Based Learning. In: J. Siekmann (Editor), Lecture Notes in Artificial Intelligence. Analogical and Inductive Inference. Int. Workshop All'89, 1-6 Octobcr 1989, Reinhardsbrunn Castle, GDR, Springer, pp. 184-201.

Salzberg, S., 1991. A nearest hyper-rectangle learning method. Mach. Learn., 6: 251-275.

Schank, R.C. and Leake, K.B., 1989. Creativity and learning in a case-based explainer. Artif. Intell., 40: 353-385. 